\title{
Optimization of Alkaline, Acidic, Ionic Liquid and Oxidative Pretreatments for Coconut Waste Conversion into Fermentable Sugars
}

\author{
Magale K. D. Rambo, ${ }^{\circledR *, a}$ Polyana M. de Melo, ${ }^{\circledR b}{ }^{b}$ Márcia M. C. Ferreira, ${ }^{c}$ \\ Michele C. D. Rambo, ${ }^{d}$ Daniel A. Bertuol ${ }^{e}$ and Victor L. Mota ${ }^{f}$ \\ aPrograma de Pós-Graduação em Ciências Ambientais, Universidade Federal do Tocantins (UFT), \\ Campus de Palmas, 77001-090 Palmas-TO, Brazil \\ 'Programa de Pós-Graduação em Ciência e Tecnologia de Alimentos, \\ Universidade Federal do Tocantins (UFT), Campus de Palmas, 77001-090 Palmas-TO, Brazil \\ 'Laboratório de Química Teórica e Aplicada, Instituto de Química, \\ Universidade Estadual de Campinas (Unicamp), 13083-970 Campinas-SP, Brazil \\ ${ }^{d}$ Instituto Federal do Tocantins (IFTO), Campus de Palmas, 77021-090 Palmas-TO, Brazil \\ ${ }^{e}$ Laboratório de Processos Ambientais (LAPAM), Departamento de Engenharia Química, \\ Universidade Federal de Santa Maria (UFSM), 97105-900 Santa Maria-RS, Brazil \\ ${ }^{f}$ Laboratório de Química, Universidade Federal do Tocantins (UFT), \\ Campus de Palmas, 77001-090 Palmas-TO, Brazil
}

\begin{abstract}
Cocos nucifera $\mathrm{L}$. is a palm tree of paramount importance in the food and chemistry industries, although over $50 \%$ of its biomass is discarded as waste. The aim of the study is to investigate different pretreatments in to coconut husks $(\mathrm{CH})$, based on acid, alkaline, ionic liquid (IL), and peroxidative, in order to produce fermentable sugars. Severity factors were calculated for pretreatments; values ranged from 0.3 to 1.7 for peroxide, from 0.01 to 1.4 for alkaline, from 1.4 to 2.8 for acid and from 2.0 to 3.0 for ionic liquid. Pretreatments were optimized (time and temperature) to maximize the sugar yield and to remove the total lignin after acid hydrolysis. Reducing sugar yield (70\%) was higher when $\mathrm{CH}$ waste was alkaline-pretreated for $2 \mathrm{~h}$ at $76.21{ }^{\circ} \mathrm{C}$. The highest lignin removal rate was recorded when alkaline $(21.4 \%)$ and peroxide solutions $(27.2 \%)$ were used. The IL did not increase sugar yield and was not effective in lignin removal. These outcomes were confirmed through infrared spectroscopy, whereas scanning electron microscopy showed increased biomass porosity during alkaline, acid and peroxide pretreatments. The IL showed little and non-significant changes. The crystallinity index notably increased after each pretreatment; besides, it was directly associated with sugar content.
\end{abstract}

Keywords: biomass, experimental design, response surface, sugar yield, lignin yield

\section{Introduction}

The Brazilian coconut industry operates at large scale (2.34 millions of tons of fruits) and it generates approximately 900 thousand tons of unused coconut husks $(\mathrm{CH}){ }^{1}{ }^{1}$ These husks could be converted into fermentable sugars due to their high cellulose and hemicelluloses contents, which have the potential to produce biofuels, high-value bioproducts and second-generation $(2 \mathrm{G})$ bioethanol based on pretreatments and acid/enzymatic hydrolysis. ${ }^{2-6}$

*e-mail: magalerambo@uft.edu.br
Biomass fractionation into its main components is a process of paramount importance that can be achieved through different pretreatments. ${ }^{5,6}$ Pretreatment disrupts the recalcitrance of the lignocellulosic biomass structure by separating lignin from cellulose. This process increases the proportion of amorphous cellulose, since it is more susceptible to acid/enzymatic hydrolysis, changes intra and inter-molecular hydrogen bonds, and enables biomass dissolution. ${ }^{7}$

Hydrolysis yield is estimated at $20 \%$ of the theoretical value when no pretreatment is adopted. ${ }^{8}$ However, many methods (steam explosion, ammonia fiber explosion, alkali 
and acid solutions, organosolv process, and wet oxidation in combination with alkaline hydrolysis) have led to high sugar yield (above 90\%). ${ }^{9}$

Literature describes several pretreatments based on dilute acid, ${ }^{10,11}$ dilute alkaline, ${ }^{3,7}$ as well as on oxidative ${ }^{12}$ and ionic liquid. ${ }^{13,14}$ Thus, it is necessary analyzing pollution-free and economically feasible methods capable of shortening the duration and reducing the costs of the process before selecting the best pretreatment. ${ }^{10}$

Overall, the success of any pretreatment depends on the adopted conditions such as temperature, time, biomass loading, and concentration. ${ }^{6}, 15$ Therefore, the aim of the current study was to optimize (temperature and time) pretreatments, based on the response surface methodology (RSM), in order to identify the process conditions capable of maximizing sugar contents and removing lignin from fermentable hydrolysates. Once the process was applied to the biomass, scanning electron microscopy (SEM), $\mathrm{X}$-ray diffraction (XRD), and Fourier-transform infrared (FTIR) were used to analyze the surface morphology and crystallinity, as well as functional groups of the pretreated sample to be compared to data about the raw coconut waste.

Furthermore, this study performed a comparative analysis of process yields after the application of different pretreatments to help better understanding suitable and sustainable techniques focused on obtaining fermentable sugars with potential to produce bioproducts in biorefineries.

\section{Experimental}

\section{Materials}

\section{Feedstock}

The coconut biomass (husks) was collected (500 g) in local dumps of Palmas County-TO, Brazil. Samples were cut into small pieces and dried at $105{ }^{\circ} \mathrm{C}$ for $12 \mathrm{~h}$. Next, they were ground in a Romer micro mill (Romer Labs, São Paulo, Brazil) and sieved (48 mesh).

\section{Method}

\section{Proximate analysis}

The proximate analysis was performed based on the American Society for Testing and Materials (ASTM).

First, the ASTM D 3173-87 method $^{16}$ was used to dry the moisture content in oven, at $105 \pm 5{ }^{\circ} \mathrm{C}$ (SP 100, SP Labor, Presidende Prudente, Brazil) for $12 \mathrm{~h}$, or until reaching constant mass. The ASTM D 3174-04 method ${ }^{17}$ was used to determine the ash content in furnace
(1200 DRP7, SP Labor, Presidende Prudente, Brazil) at $600 \pm 10^{\circ} \mathrm{C}$ for $4 \mathrm{~h}$.

\section{Extraction}

The Dionex ASE 200 system (Thermo Fisher Scientific, Waltham, MA, USA) was set at 1500 PSI, $100{ }^{\circ} \mathrm{C}, 5$-min heating time, and 7-min static cycle time in order to extract $1 \mathrm{~g}$ of coconut sample using $95 \%$ aqueous ethanol mixture, based on the accelerated solvent extraction method. Three static cycles were adopted for each sample. After the extraction process was over, the remaining solid was transferred to a Petri dish and left to air dry for 2 days to determine the extractive contents. Biomass extractions were performed in duplicate, to achieve less than $1.5 \%$ variation.

\section{Pretreatments}

The biomass pretreatments were performed to determine both sugar conversion and lignin content. The pretreated biomass yield (BY) was defined through equation 1.

Pretreated biomass yield $(\%)=\frac{\text { pretreated biomass mass }(\mathrm{g})}{\text { raw biomass mass }(\mathrm{g})} \times 100$

In addition, the severity factor was calculated based on the severity of the potential failure effect. This factor is used to compare, evaluate and predict the solubilization of biomass components. The severity factor (SF) was calculated through equation $2:{ }^{18}$

$\log \left(\mathrm{r}_{0}\right)=\log \Sigma_{\mathrm{i}}^{\mathrm{n}} 1\left[\mathrm{t} \times \exp \left(\frac{\mathrm{T}_{\mathrm{i}}-100}{14.75}\right)\right]$

wherein: $\log \left(\mathrm{r}_{0}\right)$ is severity factor; $\mathrm{t}$ : is treatment time in min; $\mathrm{T}_{\mathrm{i}}$ : is treatment temperature; 100 is reference temperature. The adjusted value 14.75 corresponds to the arbitrary constant based on the kinetic activation energy.

\section{Hydrogen peroxide pretreatment}

Hydrogen peroxide pretreatments were conducted in Erlenmeyer flasks at 1:20 solid/liquid (s/l) ratio by using 3\% $\mathrm{H}_{2} \mathrm{O}_{2}$ solution during stirring. The process was optimized for different temperatures and time reactions, based on experimental design (ED) in Table 1.

\section{Alkaline pretreatment}

Five grams of dried and milled samples were transferred to $100-\mathrm{mL}$ glass bottles and mixed with $4 \% \mathrm{NaOH}$ solutions $(\% \mathrm{~m} / \mathrm{v})$ in $50 \mathrm{~mL}$ total reaction volume to enable the alkaline pretreatment. Homogenized samples were kept in stirrers (time and temperature met the experimental design shown in Table 2) and left to cool down to room 
Table 1. Peroxide pretreatment factors and experimental design

\begin{tabular}{|c|c|c|c|c|c|c|}
\hline & Variable & -1.41 & -1 & 0 & +1 & +1.41 \\
\hline A & temperature $/{ }^{\circ} \mathrm{C}$ & 33.79 & 40 & 55 & 70 & 76.21 \\
\hline B & time / h & 2.59 & 3 & 4 & 5 & 5.41 \\
\hline Trial & A & $\mathrm{B}$ & Sugar $/ \%$ & KL $/ \%$ & ASL / \% & $\mathrm{BY} / \%$ \\
\hline 1 & -1 & -1 & 60.1 & 24.8 & 0.53 & 66.0 \\
\hline 2 & 1 & -1 & 50.5 & 26.6 & 0.46 & 62.1 \\
\hline 3 & -1 & 1 & 56.2 & 26.9 & 0.95 & 61.3 \\
\hline 4 & 1 & 1 & 41.5 & 28.2 & 0.66 & 62.0 \\
\hline 5 & -1.41 & -1 & 59.0 & 28.5 & 0.29 & 64.2 \\
\hline 6 & 1.41 & 0 & 50.8 & 30.7 & 0.40 & 61.6 \\
\hline 7 & 0 & -1.41 & 50.3 & 28.0 & 0.35 & 65.9 \\
\hline 8 & 0 & 1.41 & 38.1 & 33.3 & 0.41 & 55.6 \\
\hline 9 & 0 & 0 & 46.9 & 20.8 & 1.21 & 60.8 \\
\hline 10 & 0 & 0 & 39.5 & 21.1 & 1.31 & 63.0 \\
\hline 11 & 0 & 0 & 40.8 & 23.4 & 1.19 & 63.0 \\
\hline
\end{tabular}

A: temperature; B: time; KL: Klason lignin; ASL: acid soluble lignin; BY: biomass yield.

Table 2. Alkaline pretreatment factors and experimental design

\begin{tabular}{|c|c|c|c|c|c|c|}
\hline & Variable & -1.41 & -1 & 0 & +1 & +1.41 \\
\hline A & temperature $/{ }^{\circ} \mathrm{C}$ & 33.79 & 40 & 55 & 70 & 76.21 \\
\hline B & time / h & 0.59 & 1 & 2 & 3 & 3.41 \\
\hline Trial & $\mathrm{A}$ & $\mathrm{B}$ & Sugar $/ \%$ & KL $/ \%$ & ASL / \% & $\mathrm{BY} / \%$ \\
\hline 1 & -1 & -1 & 58.5 & 25.52 & 1.63 & 52.0 \\
\hline 2 & 1 & -1 & 62.2 & 29.76 & 1.60 & 44.1 \\
\hline 3 & -1 & 1 & 64.0 & 16.84 & 0.57 & 52.3 \\
\hline 4 & 1 & 1 & 69.0 & 14.39 & 0.47 & 40.1 \\
\hline 5 & -1.41 & 0 & 63.0 & 22.75 & 0.36 & 56.4 \\
\hline 6 & 1.41 & 0 & 70.1 & 22.83 & 0.41 & 35.1 \\
\hline 7 & 0 & -1.41 & 48.2 & 22.24 & 1.38 & 77.1 \\
\hline 8 & 0 & 1.41 & 56.0 & 17.02 & 0.38 & 56.4 \\
\hline 9 & 0 & 0 & 64.0 & 18.11 & 1.46 & 47.0 \\
\hline 10 & 0 & 0 & 59.0 & 18.11 & 1.42 & 50.0 \\
\hline 11 & 0 & 0 & 61.3 & 17.43 & 1.89 & 46.0 \\
\hline
\end{tabular}

A: temperature; B: time; KL: Klason lignin; ASL: acid soluble lignin; BY: biomass yield.

temperature. Soluble and solid (insoluble) fractions were separated based on gravimetric filtration. The solid fraction was washed with deionized water to reach $\mathrm{pH} 7$; next, it was oven dried at $45{ }^{\circ} \mathrm{C}$ and stored in plastic bags for further analysis. ${ }^{19}$

\section{Sulfuric acid pretreatment}

Samples subjected to sulfuric acid pretreatment were autoclaved (time and temperature met the experimental design shown in Table 3) using $2.5 \% \mathrm{H}_{2} \mathrm{SO}_{4}$ solution at $1: 20 \mathrm{~s} / 1$ ratio. After the autoclaving process was over, the solution was cooled down to room temperature and vacuum filtered. The solid fraction was washed with deionized water to reach $\mathrm{pH} 5,{ }^{19}$ oven dried at $45^{\circ} \mathrm{C}$, and stored in plastic bags for further analysis.
Ionic liquid (IL) pretreatment

With respect to the ionic liquid pretreatment, 1-ethyl3-methylimidazolium chloride ([EMIM]Cl) was added to the coconut biomass in a $25-\mathrm{mL}$ Erlenmeyer flask, at a final concentration of $1: 20 \% \mathrm{~m} / \mathrm{v}$ (biomass/IL). The coconut/IL mixtures were heated for different periods of time $(2,4$ and $6 \mathrm{~h})$ at different temperatures $(100,120$ and $140{ }^{\circ} \mathrm{C}$ ). After the pretreatment was over, $10 \mathrm{~mL}$ of deionized water (antisolvent) was slowly added to the mixture to precipitate the regenerated biomass; next, the solution was centrifuged. After vacuum filtration, the solid fraction was washed again with deionized water $(500 \mathrm{~mL})$ to remove excessive IL. The pretreated material was oven-dried overnight at $70{ }^{\circ} \mathrm{C}$. 
Table 3. Sulfuric acid pretreatment factors and experimental design

\begin{tabular}{|c|c|c|c|c|c|c|}
\hline & Variable & -1.41 & -1 & 0 & +1 & +1.41 \\
\hline A & temperature $/{ }^{\circ} \mathrm{C}$ & 105.8 & 110 & 120 & 130 & 134.1 \\
\hline $\mathrm{B}$ & time $/ \mathrm{h}$ & 0.29 & 0.5 & 1 & 1.5 & 2.01 \\
\hline Trial & $\mathrm{A}$ & $\mathrm{B}$ & Sugar $/ \%$ & KL / \% & ASL / \% & $\mathrm{BY} / \%$ \\
\hline 1 & -1 & -1 & 14.4 & 33.7 & 2.76 & 54.0 \\
\hline 2 & 1 & -1 & 12.6 & 35.9 & 2.98 & 50.1 \\
\hline 3 & -1 & 1 & 12.5 & 37.4 & 2.81 & 52.3 \\
\hline 4 & 1 & 1 & 25.5 & 33.6 & 2.65 & 49.0 \\
\hline 5 & -1.41 & 0 & 47.9 & 42.3 & 0.41 & 52.8 \\
\hline 6 & 1.41 & 0 & 55.5 & 39.0 & 0.25 & 55.8 \\
\hline 7 & 0 & -1.41 & 48.0 & 39.2 & 0.33 & 51.0 \\
\hline 8 & 0 & 1.41 & 58.1 & 44.1 & 0.41 & 46.9 \\
\hline 9 & 0 & 0 & 29.0 & 35.6 & 3.35 & 52.1 \\
\hline 10 & 0 & 0 & 31.0 & 34.0 & 3.27 & 52.0 \\
\hline 11 & 0 & 0 & 39.3 & 38.5 & 3.11 & 51.9 \\
\hline
\end{tabular}

A: temperature; B: time; KL: Klason lignin; ASL: acid soluble lignin; BY: biomass yield.

\section{Acid hydrolysis}

Raw and pretreated samples $(300 \mathrm{mg}$ ) were subjected to a two-stage acid hydrolysis, as described by the National Renewable Energy Laboratory. ${ }^{20}$ In the first stage, the sample and $72 \%$ sulfuric acid $(3 \mathrm{~mL})$ were subjected to water bath at $30{ }^{\circ} \mathrm{C}$ for $1 \mathrm{~h}$ and stirred every $10 \mathrm{~min}$. In the second stage, $84 \mathrm{~mL}$ of water was added to the sample, which was autoclaved at $120^{\circ} \mathrm{C}$ for $1 \mathrm{~h}$.

\section{Carbohydrate content}

The hydrolysate was added to 3,5-dinitrosalicylic acid (DNS) solution (150 in $2850 \mu \mathrm{L}$ ), boiled for $5 \mathrm{~min}$ and cooled in ice-water bath. The absorbance of reducing sugars at $540 \mathrm{~nm}$ was measured in UV-Vis spectrophotometer (Varian, Cary 4000, Berlin, Germany) in order to quantify the sugar content. Total reducing sugar concentrations were calculated based on the standard curve of D-glucose, whereas sugar conversion was determined through equation $3 .^{21}$

Sugar conversion $(\%)=\frac{\text { mass of total reducing sugars }(\mathrm{g})}{\text { mass of regenerated biomass }(\mathrm{g})} \times 100$

A test kit (LABTEST, Minas Gerais, Brazil) was used to measure the enzyme activity based on the amount of glucose formed by the glucose oxidase enzyme (GOD/POD), which is the flavoprotein used in these kits. The reaction occurring can be modified to:

$$
\begin{aligned}
& \text { Glucose }+\mathrm{O}_{2} \stackrel{\text { GOD }}{\longrightarrow} \text { gluconic acid }+\mathrm{H}_{2} \mathrm{O} \\
& 2 \mathrm{H}_{2} \mathrm{O}_{2}+\text { Phenol }+ \text { 4-Aminoantipyrine } \stackrel{\text { POD (Peroxidade) }}{\longrightarrow} \text { quinone }+4 \mathrm{H}_{2} \mathrm{O}
\end{aligned}
$$

The analytical procedure consisted in adding $1.0 \mathrm{~mL}$ of hydrolyzed $\mathrm{CH}$ sample to $1.0 \mathrm{~mL}$ of the enzyme glucose reagent solution. The same procedure was applied to standard glucose solution. The generated aliquots were subjected to water bath $\left(37^{\circ} \mathrm{C}\right)$ for $15 \mathrm{~min} .{ }^{22}$ Subsequently, sample absorbance at $500 \mathrm{~nm}$ was measured in spectrophotometer (HACH; Mod.: DR5000, Berlin, Germany).

Glucose concentration was expressed in $\mathrm{mg} \mathrm{dL}^{-1}$ based on the sample/standard absorbance ratio.

\section{Lignin content}

Klason lignin (KL) and acid soluble lignin (ASL) contents in the pretreated biomass were quantified by following the laboratory analytical procedure (NREL). ${ }^{20}$ Based on KL determination, solids retained in filter crucibles must be heated to $105^{\circ} \mathrm{C}$ for acid insoluble residue (AIR) analysis and, then, to $575{ }^{\circ} \mathrm{C}$ for acid-insoluble ash (AIA) measurement purposes. KL was determined based on the difference between AIR and AIA. ASL was analyzed in UV-Vis spectrophotometer (HACH; Mod.: DR5000, Berlin, Germany at $294 \mathrm{~nm}$ wavelength); total lignin (TL) was calculated based on the sum of KL and ASL.

The lignin content left in the pretreated biomass (\%) was defined through equation 6 :

$$
\text { Lignin content }(\%)=\frac{\begin{array}{l}
\text { mass of lignin remaining } \\
\text { in pretreated biomass }(\mathrm{g})
\end{array}}{\begin{array}{c}
\text { mass of lignin remaining } \\
\text { in raw biomass }(\mathrm{g})
\end{array}} \times 100
$$

\section{X-ray powder diffraction (XRD)}

Raw and pretreated samples were dried at $40{ }^{\circ} \mathrm{C}$ and, then, they were characterized in $\mathrm{X}$-ray diffractometer (Bruker D8 Advance, Karlsruhe, Germany) with $\mathrm{Cu} \mathrm{K \alpha}$ 
radiation and $\mathrm{k}=0.15405 \mathrm{~nm}$. Samples were scanned from 5 to $35^{\circ}(2 \theta)$ at 0.05 step size and $10 \mathrm{~s}$ step time. The crystallinity index (CrI) was calculated based on the method described by Segal et al. ${ }^{23}$

Infrared analysis $(\mathrm{KBr})$

The infrared spectrometer (FT-IR CARY 630, Agilent Technologies, Santa Clara, CA, USA) was set at wavelength ranging from 2500 to $15000 \mathrm{~nm}$, with $0.7 \mathrm{~nm}$ increment, and 32 scans, on average, to enable analyzing all samples at room temperature (raw and feedstock after pretreatments). Two spectra were collected for each sample and the average spectrum was used for data analysis.

\section{Scanning electron microscopy analysis (SEM)}

The morphology and physical structure of the coconut samples, before and after pretreatment, were subjected to SEM (Shimadzu SSX-550, Kyoto, Japan). Samples were dried at $60^{\circ} \mathrm{C}$ until reaching constant weight and coated on $\mathrm{Au} / \mathrm{Pd}$ film. All images were generated at $300 \times$ and $3000 \times$ magnification and observed at $10 \mathrm{kV}$.

\section{Experimental design and statistical analysis}

Response surface methodology (RSM) was used to find the optimal $\mathrm{CH}$ pretreatment conditions and to investigate the effect of variables such as time (X1) and temperature (X2) on sugar (Y1), Klason lignin (Y2), and acid soluble lignin (Y3) yield.

The total of 11 experimental runs based on the central composite rotational design (CCRD) were performed in the Protimiza software ${ }^{24}$ to optimize the pretreatment conditions. The experimental designs are presented in Tables 1, 2 and 3, which show the factors and their levels in the CCRD experimental conditions.

Analysis of variance (ANOVA) at $<0.05$ significance level and response surface plots were used to evaluate and identify significant factors and interaction effects among them, as well as to compare results between different runs. The validity of the models was determined based on Fisher distribution $(F$-test $)$ and determination coefficient $\left(\mathrm{R}^{2}\right)$ values.

\section{Results and Discussion}

The physicochemical composition of untreated dry coconut samples (99.7\% mass balance) is shown in Table 4. Carbohydrate percentage (32.2\%) favored the production of fermentable sugars, bioproducts, biofuels, among others. In addition, it was used to produce other value-added bioproducts with results similar to the ones reported in previous studies. ${ }^{25,26}$
Table 4. Composition of raw coconut husks $(\mathrm{CH})$ based on dry weight values

\begin{tabular}{lc}
\hline Parameter & Mean value / \% \\
\hline Total reducing sugars & $32.2 \pm 0.11$ \\
Glucose & $24.9 \pm 0.14$ \\
KL & $32.8 \pm 0.89$ \\
ASL & $1.25 \pm 0.21$ \\
TL & $34.0 \pm 0.44$ \\
Moisture & $7.66 \pm 0.01$ \\
Ash & $3.02 \pm 0.33$ \\
Extractives & $30.5 \pm 1.50$ \\
\hline
\end{tabular}

KL: Klason lignin; ASL: acid soluble lignin; TL: total lignin.

The intermediate lignin concentration (34\%) can be removed during appropriate pretreatments. ${ }^{22}$ Low ash and moisture contents $(<10 \%)$ indicated biomass with potential to be used in industrial biorefineries, since high ash contents affect acid hydrolysis by increasing acid consumption, whereas high moisture contents in the biomass enhance the transport, hamper the storage and reduce the calorific value of the biomass. ${ }^{25,26}$

The current study recorded high extractive content (approximately 30\%), which was similar to the one reported in the literature. ${ }^{3,25}$ However, these extractives were removed with ethanol before pretreatment applications; helped decreasing negative effects during the reactions.

Effect of pretreatments on the chemical composition of coconut husks $(\mathrm{CH})$

Biomass recovery (solid yield) higher than $45 \%$ was recorded after the application of alkaline, oxidative and acid pretreatments under all tested conditions. Severity factors calculated for the adopted pretreatments ranged from 0.3 to 1.7 (peroxide treatment), from 0.01 to 1.4 (alkaline treatment) and from 1.4 to 2.8 (acid treatment), based on data available in the literature..$^{27}$ Pretreatment temperature and duration were the major factors affecting pretreatment efficiency; other parameters such as $\mathrm{pH}$ had effect on biomatrix opening. ${ }^{27}$

Figure 1 summarizes different pretreatment processes applied to $\mathrm{CH}$ and their composition (major components) after the application of 11 experiments, based on the response surface methodology.

The sugar content in the raw biomass reached $32 \%$. However, this parameter increased after the application of all pretreatments, except for the acid pretreatment in runs 1, 2,3 , and 4 . The alkaline pretreatment enabled the best sugar 
conversion (increased by approximately $40 \%$ ), as shown in Figure 1. Pretreatments decreased lignocellulosic material recalcitrance by removing the lignin and hemicellulose and, therefore, by exposing the cellulose. ${ }^{13}$ The alkaline pretreatment primarily removed the lignin from the biomass, a fact that enhanced acid accessibility to the remaining sugars. ${ }^{28}$

The alkaline and oxidative pretreatments reduced cellulose absorption due to efficient insoluble lignin removal, which increased sugar yield. ${ }^{29}$

The alkaline pretreatment removed a large portion of lignin content (Figures 1b and 1c), whereas the acid pretreatment mainly hydrolyzed hemicelluloses. This
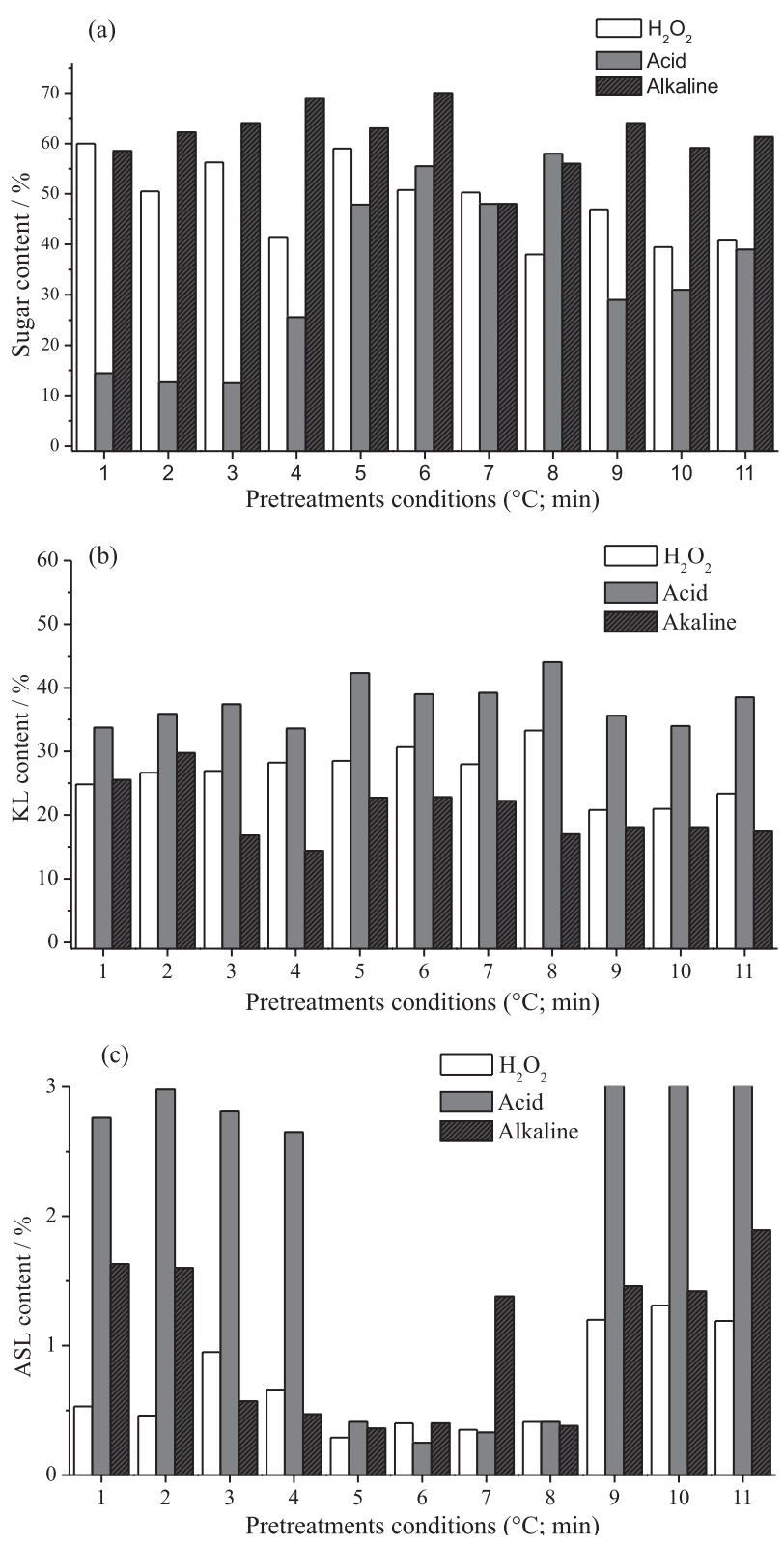

Figure 1. Effect of different pretreatments on (a) sugar, (b) Klason lignin (KL) and (c) acid soluble lignin (ASL) composition. outcome was also reported by Qing et al., ${ }^{11}$ whose acid pretreatment applied under moderate severity conditions removed over $90 \%$ hemicellulose. This pretreatment enabled reasonable sugar yield and considerable lignin content increase (ca. 10\%).

Based on the means recorded for the 11 experiments carried out in the current study, the alkaline pretreatment applied to $\mathrm{CH}$ was the most efficient in enabling higher sugar yield $(61.3 \%)$, and it was followed by the peroxide $(50.2 \%)$ and acid $(49.8 \%)$ pretreatments, as shown in Figure 1a. Similar results were reported by Shimizu et al., ${ }^{12}$ who conducted a study with banana pseudostem and reported increased glucose yield and cellulose accessibility after the acid and alkali pretreatments, whereas the peroxide pretreatment removed hemicellulose and lignin from banana samples.

Patiño et al. $^{30}$ applied an acid/alkaline-peroxide pretreatment to olive tree biomass for bioethanol production and achieved $80 \%$ delignification and $72 \%$ cellulose yield. Qing et al. ${ }^{11}$ also compared alkaline and acid pretreatments applied to soybean wastes to produce fermentable sugars. Based on their results, the acid pretreatment led to the highest total sugar yield (higher than 70\%).

Ding et al. ${ }^{31}$ also compared three different pretreatments applied to Malaysian coconut husk $(\mathrm{CH})$ to obtain fermentable sugars. Based on microwave assisted alkaline (MAA) pretreatment, the aforementioned researchers found the best conditions, which increased cellulose contents from $18-21 \%$ to $38-39 \%$, whereas lignin content decreased from 46 to $31 \%$. Acid and alkaline pretreatments increased cellulose contents 4 and $15.5 \%$, respectively. Thermal pretreatment did not show significant changes in this parameter. In comparison to the present study, acid and alkaline pretreatments recorded the best results for CH biomass results (20 and 40\%, respectively) in lesser time ( $3 \mathrm{~h}$ versus $24 \mathrm{~h}$ ). Comparing the MAA pretreatment with the present work, we obtain best yields with simple pretreatments, cheapest and less polluting.

The IL pretreatment comprising 6, 4, and 2 reaction hours enabled 27,40 , and $65.5 \%$ biomass yield, respectively. The low biomass yield recorded under reaction condition $6 \mathrm{~h}$ at $140{ }^{\circ} \mathrm{C}$ did not enable a reasonable sample for hydrolysis purposes, as shown in Figure 2 (no data about hydrolysis product). Severity factor ranged from 2.0 to 3.0 ; the highest severity value was recorded for the most severe condition.

Acid hydrolysis was used to determine the chemical composition of the biomass after IL pretreatment application (Figure 2).

The IL was not effective in extracting lignin, in increasing the accessible surface area or in dissolving carbohydrates (Figure 2). Similar findings were reported 


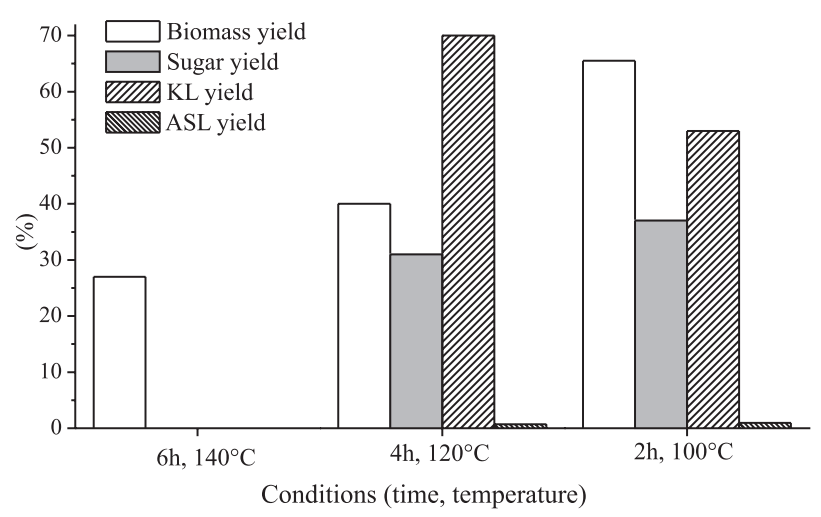

Figure 2. Effect of IL pretreatment on biomass recovery, as well as on sugar and lignin compositions.

by Torr et al.,$^{32}$ who did not observe changes in cellulose crystallinity and in lignin removal.

Based on all pretreatments, it is possible stating that alkali pretreatment processes use lower temperatures and pressures than other technologies; besides, they can be carried out under ambient conditions, within some hours. In comparison to acid processes, for example, alkaline processes cause less sugar degradation and most salts can be recovered and/or regenerated. ${ }^{9}$

However, the use of sulfuric acid concentrations lower than $4 \mathrm{wt} . \%$ is an interesting alternative due its low cost and effectiveness. Therefore, it is extremely important evaluating appropriate dilute-acid pretreatments, since the use of acid results in costly building materials, in high pressures, as well as in hydrolysate neutralization and conditioning. ${ }^{9}$

Hydrogen peroxide was a satisfactory pretreatment, since it operated under moderate temperature and time conditions, and enabled high sugar yields (> 50\%). In addition, it is a typical environmentally-friendly compound that does not leave residues. ${ }^{33,34}$ On the other hand, the ionic liquid pretreatment adopted in the present study was not effective, since it enabled low sugar yields and low lignin removal, besides being expensive and hard to be recovered.

\section{Optimization of pretreatments and development of models}

Experimental results were visualized in threedimensional response surface plots, which showed the effects of the interaction between variables "temperature" and "time" on sugar yield in pretreatments applied to lignocellulosic biomass.

Figure $3 \mathrm{a}$ shows the effects of time and temperature on sugar contents after the alkaline pretreatment. The application of high temperature increased the carbohydrate solubility associated with intermediate $(2.5$ and $3 \mathrm{~h})$ reaction times.

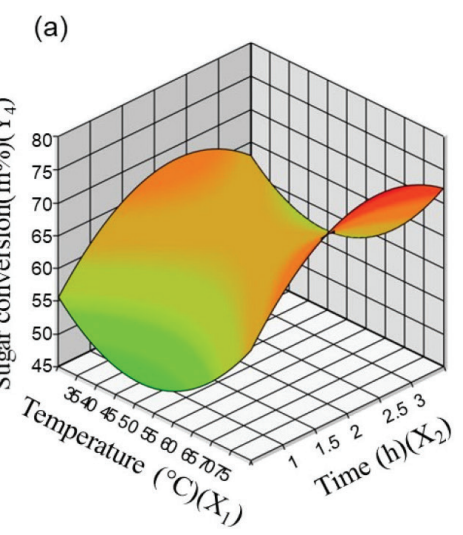

(b)

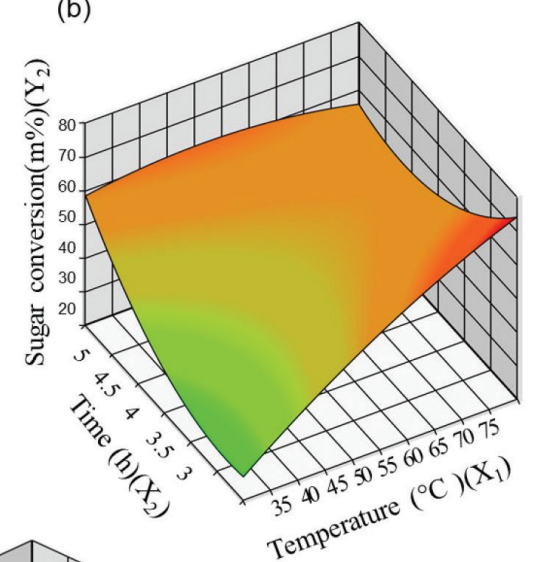

(c)

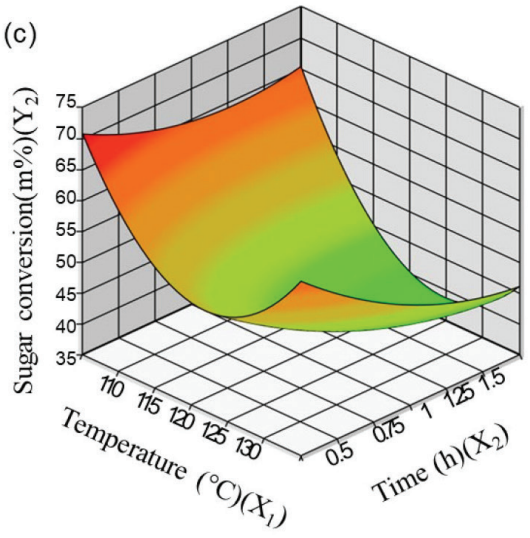

Figure 3. Response surface plot of the effect of (a) alkaline, (b) $\mathrm{H}_{2} \mathrm{O}_{2}$, and (c) acid pretreatments on total sugar contents. 
Based on the interaction between temperature and reaction time, Figure $3 b$ shows that the maximum sugar yield can be enabled by peroxide pretreatments applied at temperatures higher than $70{ }^{\circ} \mathrm{C}$, in combination with short reaction times such as $3.5 \mathrm{~h}$ and $4 \mathrm{~h}$. Only variable "temperature" has significantly influenced sugar yield in the present study. The response surface shape in Figure 3c is opposite from that shown in Figures $3 a$ and $3 b$, when the application of lower temperatures and shorter reaction times during the acid pretreatment increased sugar contents.

Figure 4 shows the effect of alkali and peroxide pretreatments on Klason lignin (Figures $4 \mathrm{a}$ and $4 \mathrm{~b}$ ) and soluble lignin contents (Figures $4 \mathrm{c}$ and $4 \mathrm{~d}$ ).

Therefore, the Klason lignin response in Figure $4 \mathrm{a}$ increased due to increased temperature $\left(>70{ }^{\circ} \mathrm{C}\right)$ in the alkaline pretreatment. However, the reaction time did not show interaction effect. Both variables showed interaction in the ASL response, as shown in Figure 4c. Reaction time had slightly negative effect on ASL at the highest temperatures.

Conversely, the oxidative pretreatment had timetemperature interaction effect on the KL and ASL (Figures $4 \mathrm{~b}$ and $4 \mathrm{~d}$ ) plots. There were positive timetemperature interactions when ASL was subjected to intermediate conditions, i.e., when the temperature was kept at $55^{\circ} \mathrm{C}$ and the adopted reaction time was $4 \mathrm{~h}$. However, KL has shown opposite results, since the best KL yield was recorded under the lowest and highest conditions.

Table 5 shows analysis of variance (ANOVA) data. High coefficient of determination $\left(\mathrm{R}^{2}\right)$ values (for $\mathrm{KL}$ and ASL in the acid pretreatment) have indicated that the herein developed models can present reliable response estimates within the range of conditions in the adopted process, ${ }^{11}$ as well as good correlation between the observed and predicted values.

The $F_{\text {values }}$ were higher than the tabulated $F_{\text {values }}$ $(F 0.0005)$ in most models, thus suggesting good prediction models. This outcome indicated that the model was significant and presented high confidence level (95.00\%). The models had significant effects, since $P$ value was lower than 0.05 , which implied $0.05 \%$ chance of having the models working due to noise. ${ }^{28}$

\section{Raw and pretreated biomass characterization}

Biomass crystallinity is a significant disadvantage for potential applications in bioconversion processes. ${ }^{35}$ It happens because cellulose is mostly distributed in two domains, namely: a highly ordered domain (crystal region) and a reasonably less ordered one (amorphous region). ${ }^{36}$
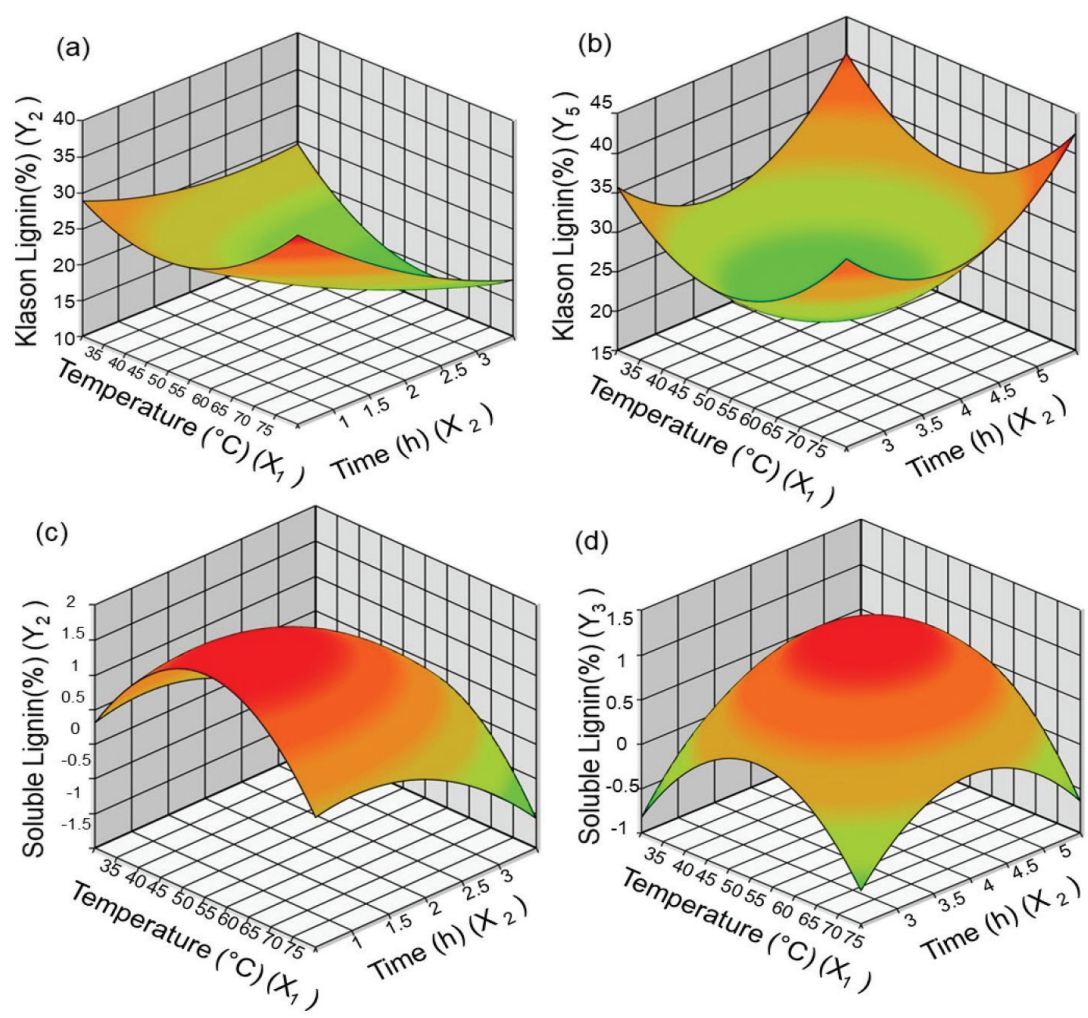

Figure 4. Response surface plot of the effect of (a) alkaline and (b) $\mathrm{H}_{2} \mathrm{O}_{2}$ pretreatments on Klason lignin (KL); as well as of (c) alkaline and (d) $\mathrm{H}_{2} \mathrm{O}_{2}$ pretreatments on acid soluble lignin contents. 
Table 5. Analysis of variance (ANOVA) for quadratic models

\begin{tabular}{|c|c|c|c|c|c|c|}
\hline Parameter & Pretreatment & $\mathrm{R}^{2}$ & $F_{\text {value }}$ & $F_{\text {tabulated } 0.05}$ & $P_{\text {value }}$ & Meaningfulness \\
\hline Sugar conversion & $\mathrm{H}_{2} \mathrm{O}_{2}$ & 0.843 & 5.4 & & 0.044 & significant \\
\hline Sugar conversion & alkaline & 0.837 & 5.1 & & 0.048 & significant \\
\hline Sugar conversion & acid & 0.890 & 8.1 & & 0.019 & significant \\
\hline KL & $\mathrm{H}_{2} \mathrm{O}_{2}$ & 0.838 & 5.2 & & 0.047 & significant \\
\hline KL & alkaline & 0.836 & 5.1 & 5.05 & 0.048 & significant \\
\hline $\mathrm{KL}$ & acid & $<0.50^{\mathrm{a}}$ & & & & non-significant $^{\mathrm{a}}$ \\
\hline ASL & $\mathrm{H}_{2} \mathrm{O}_{2}$ & 0.718 & 5.0 & & 0.050 & significant \\
\hline ASL & alkaline & 0.837 & 5.1 & & 0.048 & significant \\
\hline ASL & acid & $<0.50^{\mathrm{a}}$ & _- & & & non-significant ${ }^{\mathrm{a}}$ \\
\hline
\end{tabular}

alues are non-significant; KL: Klason lignin; ASL: acid soluble lignin; $\mathrm{R}^{2}$ : coefficient of determination.

The coconut XDR spectra before and after pretreatment application showed approximately $42 \%$ of crystalline content in the untreated biomass (Figure 5). The coconut subjected to pretreatment with $\mathrm{H}_{2} \mathrm{O}_{2}$ and acid solution presented remarkably different $\mathrm{X}$-ray diffraction intensity in comparison to the untreated coconut fiber (approximately 56 and 53\%, respectively).

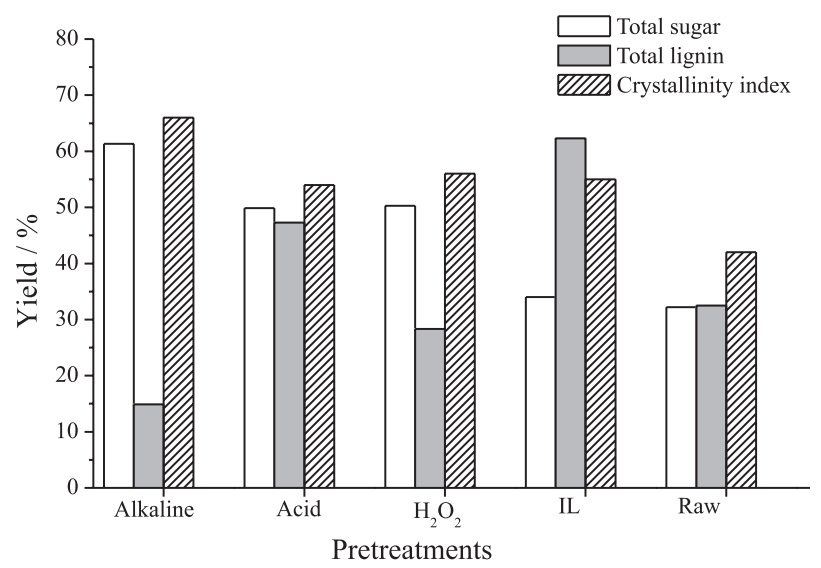

Figure 5. Effect of different pretreatments on sugar conversion, total lignin content and crystallinity index.

The crystallinity index was directly proportional to sugar conversion, as shown in Figure 5. The association between increased crystallinity index and increased sugar conversion was in compliance with the order of the following pretreatments: alkaline $>$ peroxide $>$ IL $>$ acid.

The alkaline pretreatment resulted in higher biomass crystallinity levels (66\%). This pretreatment increased biomass crystallinity by disrupting the intra- and intermolecular hydrogen bonds in the cellulose. ${ }^{32}$ Such increase indicated that the alkaline pretreatment affected amorphous cellulose solubilization in comparison to cellulose in its crystalline form. ${ }^{14}$ The increase in sugar yield for this pretreatment may be justified by the fact that non-cellulosic components have been degraded, such as hemicellulose and lignin, explained by the high crystallinity index obtained.
The chemical functional groups were analyzed based on the application of FTIR to the untreated and pretreated samples (Figure 6). Effective pretreatments were supposed to induce substantial changes in biomass components; such changes could be seen in functional-group variations.

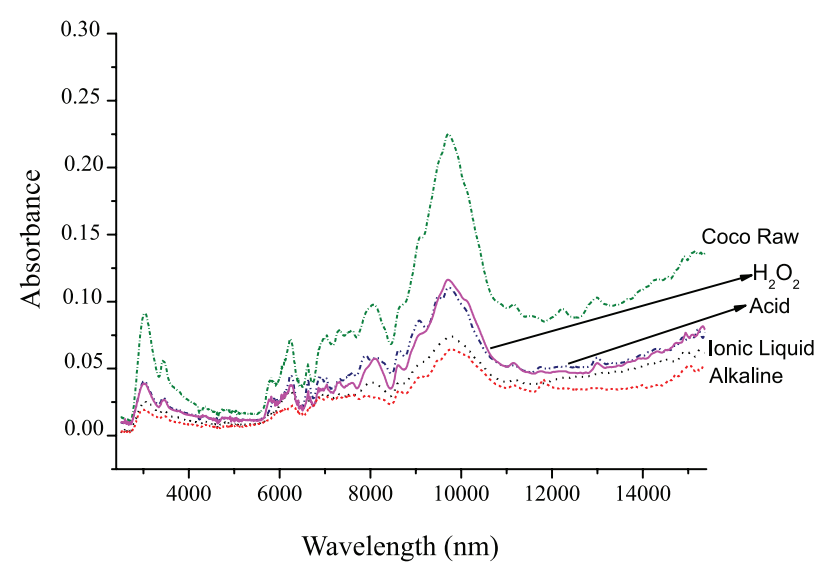

Figure 6. FTIR (KBr) spectra of the raw and pretreated samples.

The spectral characteristics of the functional groups showed substantial changes in the raw and pretreated $\mathrm{CH}$. The strongest absorption band (at wavelength $9700 \mathrm{~nm}$ ) was observed in all spectra. This outcome indicated $\mathrm{C}-\mathrm{O}$ stretching vibration in primary alcohols associated with lignin, ${ }^{37}$ since it abruptly decreased after alkaline pretreatment application, as shown in Figure 1b. Intensity peaks at 6289 and $6666 \mathrm{~nm}$ were characteristic of aromatic $\mathrm{C}=\mathrm{C}$ stretches in lignin structures; they also decreased under alkaline condition.

Another strong band (at wavelength of approximately $3000 \mathrm{~nm}$ ) was attributed to the (OH stretching) H-bond hydroxyl, which indicated H-bond disruptions in the hemicellulose, ${ }^{38}$ explained the higher absorption in the raw spectrum and changed after pretreatments. This outcome was likely associated with decreased hemicellulose fraction after the application of the most severe conditions. 
The decreasing intensity peak at wavelength $5813 \mathrm{~nm}$ was associated with acetyl groups $(\mathrm{C}=\mathrm{O}$ stretching vibration) from the hemicelluloses. Therefore, it is possible attributing the loss of these components to the most severe pretreatments.

Lower absorption bands were recorded for acid, alkaline, liquid ionic and peroxide pretreatments. This outcome was associated with lignin removal after the application of these pretreatments.

The structure and morphology of the untreated biomass, as well as the biomass pretreated with several solvents, were examined through SEM micrographs (Figure 7), which showed structural changes in them.

(a)

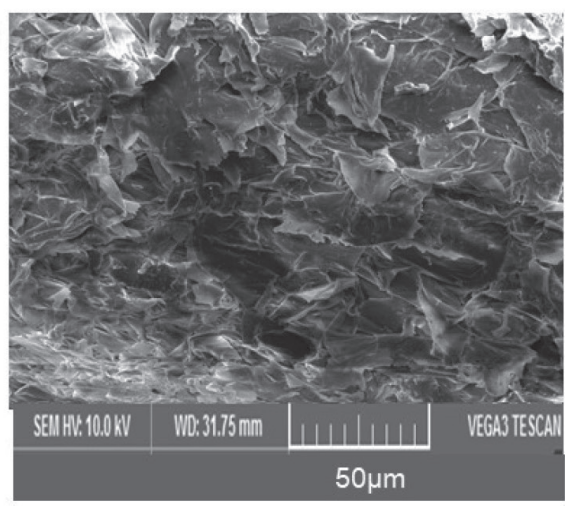

(c)

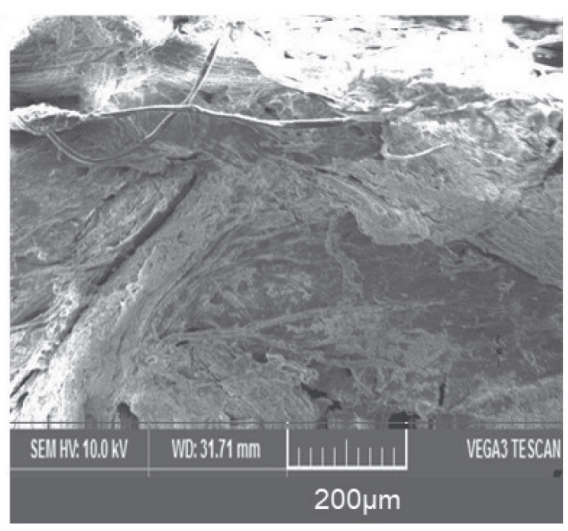

Based on SEM images, the untreated CH (Figure 7a) showed ordered and smooth structure with undamaged fiber organization. Samples pretreated with ionic liquid (Figure $7 b$ ) slowly presented morphology changes in comparison to raw samples.

Changes in the structure and morphology of biomasses subjected to the alkaline and acid pretreatments were visible (Figures $7 \mathrm{c}$ and 7d). This outcome indicated that part of the lignin fraction was removed from the fiber, since lignin is responsible for the highly compact structure of plant cell walls. $^{13}$

CH surface morphology considerably changed after the application of peroxide pretreatments; its structure was

(b)

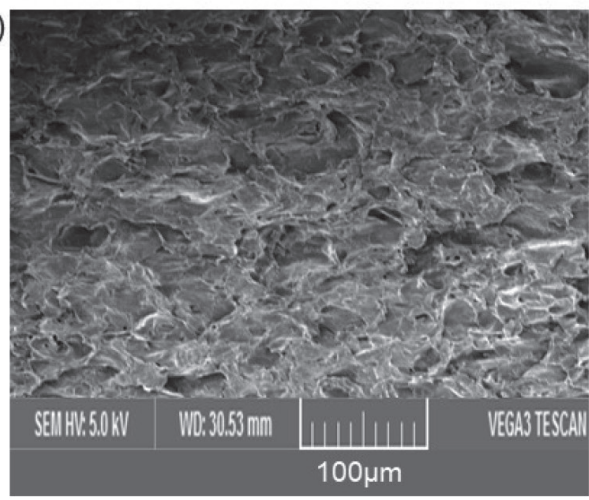

(d)

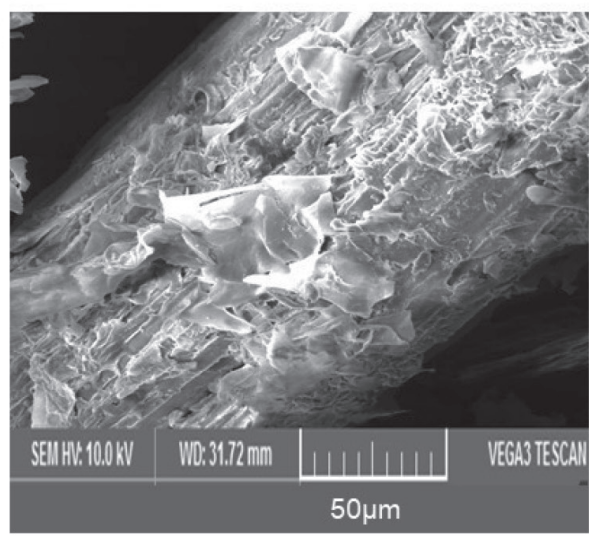

(e)

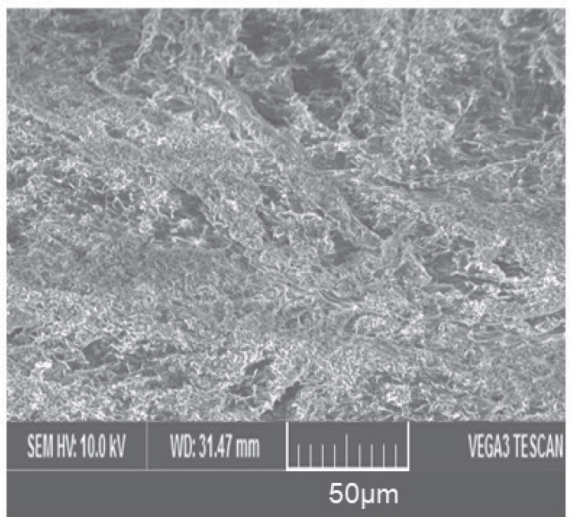

Figure 7. SEM images of (a) untreated coconut husk samples, as well as of samples subjected to (b) ionic liquid, (c) acid, (d) alkaline and (e) $\mathrm{H}_{2} \mathrm{O}_{2}$ pretreatments. 
broken and became more porous (Figure 7e). Cellulose may have been extracted from $\mathrm{CH}$ bundles during the IL pretreatment. $\mathrm{CH}$ structure was broken and became loose and porous, thus facilitating the exposure of contact locations inside or on the surface of cellulose fibers, which presented more amorphous regions in comparison to the untreated $\mathrm{CH}^{37,39}$

\section{Conclusions}

Lignocellulosic coconut husks are a prominent agroindustrial byproduct that can be beneficial to the production of value-added conversion products due to high concentrations of fermentable sugars. The pretreatments increased by three times the reducing sugar yield $(70.0 \%)$ of $\mathrm{CH}$ samples in comparison to coconut samples that were not subjected to pretreatments (32.2\%).

Statistical approaches have shown that the herein proposed models were suitable and provide a good description of the correlation between variables and response $\left(\mathrm{R}^{2}>0.83\right)$, except for ASL models, which recorded $\mathrm{R}^{2} 0.71$ for peroxide pretreatments, although they were seen as appropriate and accepted. The highest sugar yield and lignin removal rates were achieved in the alkaline pretreatment conducted at $70{ }^{\circ} \mathrm{C}$, with 3 reaction hours. Subsequently, the acid pretreatment reached 55\% total sugar content under the best condition, and it was followed by the treatment based on peroxide solutions. The ionic liquid pre-treatment did not increase sugar contents.

The XRD and SEM performed in the pretreatments were in compliance with the FTIR spectrum, which often shows organized structure in the raw lignocellulosic biomass. The chemical structure of the samples changed after the application of the herein adopted pretreatments; the crystallinity index increased based on pretreatment severity, whereas polymerization degree decreased and cellulose accessibility improved.

\section{Acknowledgments}

The authors are grateful to Federal University of Tocantins (UFT) for the financial support and to Federal Institute of Tocantins (IFTO) (No. 30/2019) and its Research Support Program-PAP/INOVA.

\section{Author Contributions}

Magale Rambo was responsible for conceptualization, data curation, formal analysis, funding acquisition, project administration, visualization, writing original draft and writing-review and editing; Polyana Melo for the conceptualization, data curation, validation, visualization, writing original draft and writing-review and editing; Márcia M. C. Ferreira for the formal analysis, software, funding acquisition and writing original draft; Michele Rambo for the data curation, formal analysis, software, funding acquisition and writing original draft; Daniel Assumpção Bertuol for the data curation, formal analysis, software and writing original draft; Victor Mota for the data curation, validation and writing original draft.

\section{References}

1. http://www.fao.org/fileadmin/user_upload/inpho/docs/Post_ Harvest_Compendium_-_Coconut.pdf, accessed in October 2019.

2. Muharja, M.; Junianti, F.; Ranggina, D.; Nurtono, T.; Widjaja, A.; Bioresour. Technol. 2018, 249, 268.

3. Rambo, M. K. D.; Rambo, M. C. D.; Melo, P. M.; Scapin, E.; Salles, Y.; Bertuol, D. A.; Oliveira, N. M. L.; Viana, G. C. C.; J. Braz. Chem. Soc. 2020, 31, 273.

4. Zhu, S.; Yang, M.; Luo, F.; Yang, X.; Xue, Y.; BioResources 2018, 13, 3 .

5. García, S. G.; Gullón, B.; Rivas, S.; Feijoo, G.; Moreira, M. T.; J. Cleaner Prod. 2016, 120, 170.

6. Kim, J. S.; Lee, Y. Y.; Kim, T. H.; Bioresour. Technol. 2016, 199, 42.

7. Lau, B. B. Y.; Yeung, T.; Patterson, R. J.; Aldous, A. L.; ACS Sustainable Chem. Eng. 2017, 5, 5320.

8. Sánchez, Ó. J.; Cardona, C. A.; Bioresour. Technol. 2008, 99 , 5270 .

9. Kumar, P.; Barrett, D.; Delwiche, M. J.; Stroeve, P.; Ind. Eng. Chem. Res. 2009, 48, 3713.

10. Rodríguez, S. B.; Toquero, C.; Martín-Juárez, J.; Travaini, R.; García-Encina, P. A.; Bioresour. Technol. 2016, 201, 182.

11. Qing, Q.; Guo, Q.; Zhou, L.; Gao, X.; Lu, X.; Zhang, Y.; Ind. Crops Prod. 2017, 109, 391.

12. Shimizu, F. L.; Monteiro, P. Q.; Ghiraldi, P. H. C.; Melati, R. B.; Pagnocca, F. C.; Souza, W.; Sant'Anna, C.; Brienzo, M.; Ind. Crops Prod. 2018, 115, 62.

13. Uju; Goto, M.; Kamya, N.; Bioresour. Technol. 2016, $214,487$.

14. Kogut, S. M.; Zgórska, K.; Kogut, T.; Kukiełka, K.; Wojdalski, J.; Kupczyk, A.; Dróżdż, B.; Wielewska, I.; Fuel 2017, 191, 266.

15. Shah, S. S. M.; Luthfi, A. A. I.; Low, K. O.; Harun, S.; Manaf, S. F. A.; Illias, R. M.; Jahim, J. M.; Sci. Rep. 2019, 9, 4080.

16. ASTM D 3173-87: Standard Method for Determination of Moisture Content in Biomass, West Conshohocken, 2003.

17. ASTM D 3174-04: Standard Method for Ash in the Analysis Sample of Coal and Coke, West Conshohocken, 2004.

18. Overend, R. P.; Chornet, E.; Philos. Trans. R. Soc., A 1987, 321, 536. 
19. Brienzo, M.; Ferreira, S.; Vicentim, M. P.; Souza, W.; Sant'Anna, C.; BioEnergy Res. 2014, 7, 1454.

20. NREL/TP-510-42618: Determination of Structural Carbohydrates and Lignin in Biomass, Colorado, USA.

21. Weerachanchai, P.; Lee, J. M.; Bioresour. Technol. 2014, 169, 336.

22. Visvanathan, R.; Jayathilake, C.; Ruvini, L.; Food Chem. 2016, $211,853$.

23. Segal, L.; Creely, J. J.; Martin, A. E.; Conrad, C. M.; Text. Res. J. 1959, 29, 786.

24. Rodrigues, M. I.; Protimiza Experimental Design, version 2014/1; Campinas, 2014, available at http://experimentaldesign.protimiza.com.br/, accessed in October 2019.

25. Rambo, M. K. D.; Schmidt, F. L.; Ferreira, M. M. C.; Talanta 2015, 144, 696 .

26. Ram, M.; Mondal, K.; Energy 2018, 156, 122.

27. Pedersen, M.; Meyer, A. S.; New Biotechnol. 2010, $27,739$.

28. Kim, I.; Rehman, M. S. U.; Han, J. I.; Bioresour. Technol. 2014, $152,316$.

29. Seidl, P. R.; Goulart, A. K.; Curr. Opin. Green Sustainable Chem. 2016, 2, 48.

30. Patiño, J. C. M.; Ruiz, E.; Romero, I.; Cara, C.; Linares, J. C. L.; Castro, E.; Bioresour. Technol. 2017, 239, 326.
31. Ding, T. Y.; Hii, S. L.; Ong, G. A.; BioResources 2012, 7, 1540.

32. Torr, K. M.; Love, K.; Simmons, B. A.; Hill, S. J.; Biotechnol. Bioeng. 2016, 113, 540.

33. Rabelo, S. C.; Andrade, R. R.; Filho, R. M.; Costa, A. C.; Fuel 2014, 136, 349.

34. Mou, H. Y.; Heikkila, E.; Fardim, P.; Bioresour. Technol. 2013, 150,36 .

35. Weerachanchai, P.; Lee, J. M.; ACS Sustainable Chem. Eng. 2013, $1,894$.

36. Shahabazuddin, M.; Chandra, T. S.; Meena, S.; Sukumaran, R. K.; Shetty, N. P.; Mudliar, S. N.; Bioresour. Technol. 2018, 263, 199.

37. Ang, T. N.; Ngoh, G. C.; Chua, A. S. M.; Lee, M. G.; Biotechnol. Biofuels 2012, 5, 67.

38. He, Y.; Pang, Y.; Liu, Y.; Li, X.; Wang, K.; Energy Fuels 2008 , $22,2775$.

39. Cheng, J.; Zhang, J.; Lin, R.; Liu, J.; Zhang, L.; Cen, K.; Bioresour. Technol. 2017, 228, 348.

Submitted: July 15, 2019 Published online: October 31, 2019 